\title{
EL ESTUDIO DEL LENGUAJE COMO CIENCIA NATURAL
}

\author{
“... volver a colocar al hombre a la altura del \\ mundo natural más vasto, para devolverle \\ -dicho en pocas palabras-el sabor de estar \\ vivo."
}

Italo Calvino, Ermitaño en París

\section{Guillermo Lorenzo González* \\ Universidad de Oviedo}

\begin{abstract}
RESUMEN. La elevación de una propiedad del signo lingüístico (la arbitrariedad en que se basa la unión entre sus dos planos) a la categoría de propiedad general del lenguaje humano, ha motivado que el estructuralismo vaciara la investigación lingüística de cualquier género de contenido natural. En la primera parte de este artículo se propone lo que el autor llama el 'argumento de las lenguas imposibles' con el objeto de retrotraer y rebatir el razonamiento estructuralista. Este argumento deja al descubierto que la gama de lenguas particulares posibles está limitado naturalmente, lo cual justifica la emergencia de una Ciencia Natural referida al lenguaje humano. La segunda parte del trabajo se ocupa de ciertos rasgos de las modernas ciencias de la Naturaleza, y se analiza su manifestación y sus efectos en el estudio del lenguaje.
\end{abstract}

ABSTRACT. A single property of the linguistic sign (the arbitrary character of the union between its faces) has been raised as a general property of human language by structuralist linguists. This move has emptied structuralist research of any kind of natural content. In the first section of this article it is proposed what the author calls the 'argument of the impossible languages' with the aim of refuting the structuralist reasoning. This argument clearly shows that the range of particular languages is naturally limited, which justifies the emerging of a Natural Science referred to human language. The second part of the paper is devoted to certain features of modern natural sciences, whose manifestation and effects in the study of language are analyzed.

* Dr. en Filología Hispánica (Lengua). Profesor Asociado del Área de Lingüistica General del Departamento de Filología Española. Universidad de Oviedo. Campus de Humanidades "El Milán”, c/ Teniente A. Martínez, 33011 OVIEDO. E- mail: glorenzo @ vmesa.cpd.uniovi.es. 
Intentaré aclarar, antes de entrar en materia, algunos aspectos de lo expresado en el título de este trabajo, con el objeto de evitar algún prejuicio y una posible predisposición al rechazo de cuanto aquí se diga. Mi actitud personal hacia el tema que se enuncia en el título es de pleno respaldo y cuanto diga en las páginas que siguen será en apoyo de la idea que en él se expresa. Desde este punto de vista, mi título puede entenderse como una especie de 'lema' que trataré de respaldar. Me apresuro a precisar, sin embargo, que el 'como' que aparece en el título no equivale a un signo de igualdad. Quiero decir que no debe entenderse que vaya a defender que el campo de estudio de la Lingüística se agota en los aspectos 'naturales' de su objeto; tampoco que sean los más importantes en una imaginaria escala en la asignación de tareas entre sus practicantes; ni siquiera que constituyan el fin último de la investigación sobre el lenguaje. Defenderé, únicamente, que es necesario conceder una prioridad epistemológica a tales aspectos, en el sentido de que cualesquiera otros deben su forma, sus límites y hasta su misma posibilidad a las propiedades básicas del lenguaje en tanto que hecho enraizado en la naturaleza.

Consciente de que parte del interés que pueda despertar este trabajo procede del hecho de que se enfrenta a modos y hábitos propios de la atmósfera de trabajo de muchos lingüistas, en la primera parte del artículo me centraré en esta especie de tensión, tratando de explicar su origen y algunas de sus consecuencias. En la segunda parte del trabajo, superado todo tono polemizador, trataré de exponer en términos más positivos algunos de los rasgos que los estudios sobre el lenguaje están llamados a adquirir en virtud de su integración en el programa "naturalista" del que se habla en las páginas que siguen.

\section{Cultura y naturaleza en la ciencia del lenguaje}

1.1. La arbitrariedad de la cultura. Es por todos sabido que el estructuralismo de herencia saussureana ha asumido como uno de sus principios indiscutibles el carácter inequívocamente cultural de las lenguas habladas por los seres humanos (evitaré la etiqueta 'lengua natural', a mi juicio acertada y práctica, pero sin duda tendenciosa a estas alturas de la argumentación). En concreto, se dice que el objeto de una disciplina es 'cultural' si 'remite formalmente a un sujeto sin cuya existencia carecería de sentido’ -del Teso [1990: 19]-. Esta definición necesita de cierta precisión, sin la cual parece que quedaríamos autorizados para reconocer como culturales los conocimientos acerca del corazón, el hígado o cualesquiera otros órganos del cuerpo humano. Lo crucial en la anterior definición es la idea de 'remisión formal a un sujeto', la cual implica que se toma a los individuos como agentes (no necesariamente conscientes; digamos que, cuando menos, 'espontáneos') de algún género de manipulación sobre el objeto en cuestión. En el caso de la investigación lingüística, el objeto sería cultural -en palabras de Prieto [1973: xx]- porque consiste en 'el conocimiento espontáneo 
que ciertos sujetos -los hablantes- tienen de ciertos objetos'. La idea consiste, pues, en que los objetos del lenguaje no son, sin más, hechos de la naturaleza, sino hechos de la naturaleza sometidos a patrones cuya fuente inequívoca son los sujetos que los manipulan. En palabras de Bickerton (aunque tergiversando su enfoque): 'otras especies se adaptan al mundo natural; nosotros adaptamos el mundo natural a nosotros' -Bickerton [1990: 15]-. Lo específico del lenguaje sería, según este enfoque, los patrones que los hablantes superponen a los aspectos de la naturaleza de los cuales se sirve el lenguaje, patrones que deben ocupar de forma exclusiva a la disciplina encargada de la exploración lingüística.

La idea recién resumida es inseparable de (y en esencia se solapa con) la noción que fundamentalmente inspira a la investigación lingüística de tipo estructural: la idea de 'arbitrariedad'-Prieto [1973: xxi], del Teso [1990: 24]-. Es arbitrario lo que siendo de una determinada forma, podría presentar otra forma cualquiera sin que por ello peligrase su adscripción a un determinado género o clase. En este sentido, los hechos del lenguaje se nos revelan culturales en la misma medida en que comprobamos su esencial arbitrariedad, es decir, la ilimitación que se pone de manifiesto en la evidente variabilidad de los usos lingüísticos de las diferentes comunidades de hablantes, cada una de las cuales parece que proyecta un peculiar 'punto de vista' -Saussure- sobre los medios naturales que manipula el lenguaje.

El punto de vista estructural se puede resumir, en conclusión, del siguiente modo: el lenguaje no tiene otra realidad que no sea la proyección de patrones arbitrarios sobre la materia prima proporcionada por la naturaleza. Por tanto, si la realidad que llamamos lingüística no se origina hasta la proyección de esos patrones, los elementos naturales de los que se aprovecha quedan mayormente trascendidos en la elaboración linguiística y básicamente desenfocados del campo de estudio de los lingüistas.

1.2. Lenguas imposibles. Habiendo sacado a la luz los aspectos fundamentales del razonamiento estructuralista sobre el carácter cultural de los hechos lingüísticos, creo contar ya con elementos suficientes para oponerle ciertas dificultades y problemas. Más aún, para ponerlo en serios apuros. Si el carácter 'cultural' del lenguaje se desprende de su esencial 'arbitrariedad', tendríamos cierta autoridad para dudar de aquel (el 'carácter cultural') si pudiéramos poner en duda a ésta (la 'esencial arbitrariedad'), y así neutralizado el argumento, podríamos (re)abrir legítimamente el caso sobre el espacio de lo 'natural' en el estudio del lenguaje. Me propongo mostrar, con este fin, que no todo es tan arbitrario en el lenguaje como parece, e invito para ello a considerar la siguiente serie de ejemplos:

\subsection{Español}
a. _ Comerá en casa
b. Acudirá mi madre en mi lugar
c. ¿Quién dijiste que _ acudirá en tu lugar? 


\subsection{Griego}

a. Dhiavazontas tipota shetiko, _ tha katalavi perisotera leyendo algo relevante, _ fut. entender más

'Leyendo algo relevante, entenderás más'

b. Idha oti efige o Yannis

ver-1p. que se fue Yanis-nom

'Veo que se fue Yanis'

c. Pjos ipes __ oti efige?

quién-nom decir-2p. que se fue?

¿Quién dices que se fue?

\subsection{Inglés}
a. * w will eat at home
fut. comer en casa
b. * will go my mother instead of me fut. ir mi madre en lugar de mí
c. * who did you say that _ will go instead of you? quién pas. tú decir que fut. ir en lugar de mí

\subsection{Beni-Hassan (Árabe)}

a. Fariid gaal [innu __ istarat al-libaas]

Fariid dijo que compró el vestido

'Fariid dijo que se compró el vestido'

b. Fariid gaal [innu istarat al-binit al-libaas]

Fariid dijo que compró la chica el vestido

'Fariid dijo que la chica se compró el vestido'

c. wayy binit Fariid gall [innu _ istarat al-libaas]?

qué chica Fariid dijo que compró el vestido

¿QQué chica dijo Fariid que se compró el vestido?'

\subsection{Palestino (Árabe)}
a. * Fariid kaal [innu _ istarat 1-fustaan]
Fariid dijo que compró el vestido
b. * Fariid kaal [innu istarat l-bint 1-fustaan]
Fariid dijo que compró la chica el vestido
c. * ayy bint Fariid kall [innu _ istarat 1-fustaan]?
qué chica Fariid dijo que compró el vestido

En esta relación de ejemplos se ilustran tres fenómenos sintácticos diferentes: (a.) la posibilidad o no de omitir el sujeto léxico, (b.) la posibilidad o no de colocar el sujeto léxico en posición postverbal (teniendo en cuenta que consideramos únicamen- 
te lenguas en las que la posición preverbal es la 'no marcada' para los sujetos), y (c.) la posibilidad o no de realizar preguntas acerca del sujeto de una cláusula subordinada a través de una conjunción. Lo que observamos es que el valor que cada una de las lenguas consideradas adopta con relación a cada uno de los fenómenos coincide con el valor fijado para los otros dos: se aprecia, en efecto, que si una lengua fija el valor positivo para el fenómeno (a.) -esto es, sí puede omitir el sujeto léxico-, fija también el valor positivo para los fenómenos (b.) y (c.)- es decir, sí puede posponer el sujeto al verbo, y sí puede preguntar acerca del sujeto a través de una conjunción-; en cambio, si una lengua fija el valor negativo para el fenómeno (a.) -no puede omitir el sujeto léxico-, también fija negativamente los fenómenos (b.) y (c.) -no puede posponer el sujeto al verbo, y no puede preguntar acerca del sujeto a través de una conjunción-. De lo dicho resultan dos tipos lingüísticos diferentes, como se representa en el siguiente cuadro:

\begin{tabular}{|c|c|c|c|l|}
\hline & $\begin{array}{c}\text { Sujeto } \\
\text { Omitido }\end{array}$ & $\begin{array}{c}\text { Sujeto } \\
\text { PostV. }\end{array}$ & $\begin{array}{c}\text { Preguntas sobre } \\
\text { el sujeto }\end{array}$ & Ejemplos \\
\hline Tipo A & $\sqrt{ }$ & $\sqrt{ }$ & $\sqrt{ }$ & Español, Griego, Arabe Beni-Hassan \\
Tipo B & $*$ & $*$ & $*$ & Inglés, Arabe Palestino \\
\hline
\end{tabular}

Nótese que he procurado reflejar el hecho de que cada uno de los tipos engloba lenguas de filiación diversa, y que a lenguas muy próximas entre sí desde este punto de vista (variantes dialectales, de hecho) les corresponde un tipo diferente. La conclusión más interesante que cabe extraer de lo anterior es que la observación conjunta de todos estos fenómenos nos conduce a pensar en la imposibilidad de ciertos tipos de lenguas. Es una 'lengua imposible', por ejemplo, aquella en que el fenómeno (a.) se fijara positivamente y los fenómenos (b.) y (c.) negativamente -es decir, $[\sqrt{ }, *$ * *]-; en fin, cualquiera que no mostrara un valor uniforme en la fijación de los tres fenómenos. Téngase en cuenta que he seleccionado uno de los 'sistemas de fenómenos' más exhaustivamente estudiados en el marco de la lingüística generativa -el llamado, 'Parámetro del Sujeto Nulo'; mis datos proceden especialmente de Rizzi [1989], Jaeggli \& Safir [1989] y Smith \& Tsimpli [1991]-, lo cual debe dar idea de que no estoy formulando ninguna generalización con frivolidad.

El corolario que alcanzamos es la siguiente: no parece que sea posible cualquier lengua con cualesquiera características. O en otras palabras: no es el caso que fenómenos que se presentan bajo una cierta forma en una determinada lengua, hubieran podido presentarse bajo otra forma diferente. Por tanto, los márgenes de la arbitrariedad parecen estar a menudo marcados por unos estrechísimos límites, todo lo cual nos lleva a una conclusión que expresaré bajo la forma de una paradoja: hay lenguas imposibles. 
Llamaremos a lo anterior el argumento de las 'lenguas imposibles', esto es, lenguas que reúnen propiedades que en principio sería concebible que se manifestaran conjuntamente, pero que la investigación revela absolutamente incompatibles entre sí.

Con el argumento de las lenguas imposibles en nuestras manos, podemos proceder a retrotraer el razonamiento estructuralista: si la arbitrariedad de los hechos lingüísticos no es tan rotunda como en principio estábamos inclinados a creer, la propia 'culturalidad' de tales hechos queda en buena medida bajo sospecha. A partir de este momento quedamos enteramente autorizados para especular acerca de su motivación o determinación natural, que parece más profunda y que afecta al formato de las diferentes lenguas de manera mucho más decisiva y directa de lo que estábamos acostumbrados a pensar.

Llegados a este punto conviene plantearse dos cuestiones: en primer lugar, ¿cuál es la razón de ese 'totalitarismo culturalista' tan propio del estructuralismo?; en segundo lugar, aun aceptando la intromisión de la naturaleza en los hechos del lenguaje, ¿no cabría decir que su incidencia es, con todo, marginal en lo que toca a los intereses de la Linguiística? Es decir, ¿no debería ocuparse ésta únicamente de lo que cae dentro de lo limitado naturalmente, y en ningún caso los límites mismos?

1.2.1. Los excesos del signo. La respuesta a la primera cuestión se encuentra, a mi juicio, en la sobrecarga de responsabilidades que la lingüística estructural ha confiado a la idea de 'signo'. A ella confían de hecho los estructuralistas la especificidad del lenguaje humano, en el sentido de que cualquiera de los rasgos definitorios del 'signo' se generalizan automáticamente como rasgos definitorios del lenguaje: de este modo los rasgos 'esenciales' del lenguaje humano acaban por resultar los mismos que los del signo linguiístico. Un solo ejemplo: cuando Martinet proclama que el lenguaje obedece a un 'principio de doble articulación' -Martinet [1960: 22-24]-, en realidad nos está hablando de una característica del signo lingüístico; en el lenguaje, más generalmente considerado, podemos apreciar más niveles de articulación que dos.

Esta manera de razonar se aprecia bien en el trabajo ya citado de del Teso [1990] -tal vez la más completa y seria fundamentación epistemológica con que cuenta la lingüística estructural-. Así, tras haber hecho declaración del contenido exclusivamente cultural de las disciplinas lingüísticas, se apresura a razonar lo siguiente:

'No debe olvidarse que el lenguaje del que trata la lingüística es siempre un hecho cultural. Cualquier acontecimiento al que podamos llamar signo siempre lo es. En todo signo hay siempre un estímulo físico presente con el que regularmente está asociada una realidad ausente. [...] La relación dada entre los dos elementos que configuran el signo de ninguna manera puede entenderse como interna a esos elementos. No hay nada intrínseco a la experiencia acústica /mésa/ que justifique su relación con la experiencia 'mesa', ni a la inversa.'

-del Teso [1991: 19]- 
Como pueden ver, el párrafo ejemplifica absolutamente la estrategia que trato de desmontar: una propiedad del signo lingüístico -la inmotivación entre sus dos planosse eleva al rango de propiedad del lenguaje como totalidad.

No trato de cuestionar, como es lógico, el carácter inmotivado de la asociación entre los planos de expresión y de contenido de los signos lingüísticos. La cuestión, en verdad, se encuentra totalmente fuera de debate a estas alturas del pensamiento lingüístico; de hecho, es incluso improbable que Platón la cuestionara realmente, como nos recuerda en un artículo reciente Fernández de Castro [1993]. Y ya que viene a cuento este trabajo, me permitiré dirigirle también a él la crítica que estoy realizando del estructuralismo. El título del trabajo de Fernández de Castro anuncia con claridad el tema desarrollo a lo largo de sus casi cuarenta páginas : 'La Continuidad del Naturalismo Linguiístico'. Lo que aquí interesa, por sintomático, es que el único naturalismo lingüístico cuya continuidad se examina es el que intermitentemente aparece en la historia del pensamiento con relación a la asociación entre los dos planos del signo. Y no exactamente porque el autor descarte el estudio de otros posibles aspectos naturales susceptibles de ser atribuidos al lenguaje, sino más bien porque lo considera 'paradigma' de todos los posibles naturalismos. Véase en la siguiente cita:

'Más allá de las etiquetas, revisar la pervivencia del debate entre naturalismo y convencionalismo desde su borroso origen en el Crátilo, el reiterado empeño de los hombres, al reflexionar sobre el lenguaje, en afirmar que éste se halla o no se halla determinado por la naturaleza, es algo no necesario para la lingüística general desde el Curso de Saussure, pero sí conveniente para la historia de las ideas lingüísticas hasta ese mismo momento.'

-Fernández de Castro [1993: 328]; el subrayado es mío-

Me baso en esta misma cita para concluir, con relación a esta cuestión, que si el estudio de la determinación natural del lenguaje es algo de lo que la linguística se ha liberado desde Saussure, de ser cierto -como defiendo- que tal liberación deriva de un abuso de la idea de 'signo' y, más concretamente, de su posición entre los hechos del lenguaje, urgirá replantearse la necesidad y el estatuto del estudio de lo natural dentro de las disciplinas dedicadas al estudio de los fenómenos relacionados con el hablar. Esto nos lleva ya a la segunda de las preguntas que dejamos abiertas algo más arriba.

1.2.2. El desprecio del límite. Aun concediendo que la naturaleza pone límites al formato de las lenguas, ¿no deberá tratar la Lingüística de lo que cae dentro de esos límites naturales, y en ningún caso de los límites mismos? La cuestión no es simple. Escuchemos una vez más, como primer paso, a del Teso:

'No cabe afirmar que la predisposición por la que la GU (Gramática Universal que, según Chomsky, todo individuo tiene al nacer en algún lugar de 
la fisionomía de los órganos responsables de las facultades cognitivas) se va desarrollando y concretando en una lengua es hasta cierto punto semejante a la predisposición por la que al tronco le nacen brazos y piernas. Las facultades innatas determinan que ciertas manifestaciones sean posibles (andar a dos patas, hablar en español) y otras imposibles (volar agitando los brazos...). Digamos que nuestra información genética abre un abanico, pero lo cultural no está en los límites del abanico sino en su concreción.'

-del Teso [1990: 19]-

Queda claro en esta opinión que no es cuestión de negarle al lenguaje cualquier género de apoyo, asiento o soporte natural. Ni el mismo Saussure pretendió tanto. Parece más bien que Saussure y sus herederos sólo tienen interés en minimizar (idealmente, en anular) la incidencia de este soporte sobre el formato de los hechos del lenguaje -véase, no en vano, Saussure [1915: 73-75]-. La idea es que asegurados unos mínimos (el manejo de unidades biplanas, el recurso a mecanismos estables para la combinación de éstas en entidades mayores, etc. - es decir, los llamados 'universales esenciales' del lenguaje; véanse Coseriu [1978] o Hockett [1963]-), ningún dato es descartable de antemano como posible propiedad de una lengua. Además, la motivación de estos rasgos mínimos comunes a toda lengua tal vez no proceda del soporte natural encargado de su administración, sino de las propiedades del canal y de las características de su transmisión, y puede que hasta no sean específicos de las lenguas, sino comunes a todo sistema comunicativo. Saussure pensaba, de hecho, que lo naturalmente fundado no debía de ser la capacidad de poseer y manejar lenguas con unas determinadas características, sino la capacidad de generar y utilizar sistemas de comunicación -Saussure [1915: 75]-.

Es normal que un soporte natural de tan escasa como genérica incidencia sobre las propiedades de las lenguas quede descartado como tema para la curiosidad de los lingüistas. Ahora bien, recordemos que la supuesta intrascendencia del soporte resulta de la aplicación de un procedimiento de dudosa corrección: la elevación de las propiedades del signo lingüístico al rango de propiedades generales del lenguaje -lo que en la práctica equivale, por ejemplo, a elevar las propiedades de una determinada familia de arbustos al rango de propiedades generales del bosque como totalidad vegetal-.

Aquí ya se ha visto que el capricho de la naturaleza parece condicionar las propiedades del lenguaje mucho más de lo que los estructuralistas están capacitados para apreciar debido a su estrecha vigilancia de las propiedades del signo y al descuido de su atención sobre otros aspectos lingüísticos. La naturaleza, según el punto de vista defendido aquí, tiene un efecto limitador clarísimo sobre el formato de las lenguas, mucho más estrecho de lo que se supone en la tradición estructural. En este nuevo contexto de investigación, la observación de los límites adquiere una importancia tan grande como la observación de la concreción; incluso mayor -aunque esto es tal vez una cuestión de gusto y preferencia profesionales-, si tenemos en cuenta que esconden algo así como la esencia formal del lenguaje. 
El estudio del lenguaje como Ciencia Natural comienza en el momento mismo en que nos proponemos estudiar el origen de estas limitaciones como expresión de cierto género de determinismo biológico, por más que estemos ante un fenómeno en el que la naturaleza delimita, sin agotar, un campo que además queda abierto a la intervención individual (más o menos consciente) y sobre todo colectiva, camuflándolo en cierto modo entre los hechos de la cultura y de la tradición, de cuya savia es cierto que no deja de alimentarse.

Es importante señalar el giro que la investigación linguiística experimenta en la medida en que acepta la determinación natural de su objeto. Señala del Teso [1990: 28-31] que la tarea de la Lingüística General, en tanto que proveedora y supervisora de los elementos de análisis de objetos culturales, es decir, esencialmente arbitrarios, está prácticamente exenta de condicionamientos 'apriorísticos', en la medida en que la clase que trata de caracterizar -el lenguaje humano- no responde a ninguna realidad anterior a los miembros de tal clase -las lenguas concretas-. Señala, en consecuencia, que la clase que la Linguística trata de caracterizar es de tipo 'combinatorio', ya que la identidad que se establece entre los elementos de tal clase se basa 'en las partes formales genéricas que resultan de un análisis inmanente de los objetos' -del Teso [1991: 29]-. El punto de vista expuesto en este trabajo motiva una distinta atribución de funciones a la Lingüística General, y en el fondo hasta le atribuye un 'objeto concreto', que en el enfoque estructuralista no tiene. En el enfoque naturalista el lenguaje tiene una realidad subyacente -llamémosla 'Facultad del Lenguaje'- anterior a las entidades en que se nos manifiesta -las lenguas concretas-, sobre las cuales ejerce un efecto limitador; una realidad única, pues, que acaba por materializarse en entidades diversas, cuyos márgenes de variación resultan firmemente acotados por ese origen común. En este sentido, la Lingüística experimenta un giro que la lleva a hablarnos de una clase 'porfiriana', no 'combinatoria', pues a su intensión se le atribuye una existencia 'como unidad al margen de los elementos que constituyen la extensión' -del Teso [1991: 29]-.

Expresado mediante otras palabras: la Lingüística General es, en el marco del estructuralismo, una disciplina con objetivos claros, pero sin objeto; su inscripción en un programa naturalista le restituye, en cambio, un objeto de estudio definido: la facultad de la mente que limita la gama de las lenguas humanas posibles.

\section{Algunos Atributos de la Ciencia Natural del Lenguage}

2.1. Algo más sobre el 'objeto' de la Lingüística. El enfoque naturalista del lenguaje concibe a éste como un aspecto del ser humano claramente condicionado por ciertas propiedades naturales de su cerebro, caracterizado por la moderna ciencia neuro-computacional como: 
'[...] an interconnected collection of special-purpose systems that are very efficient at performing their tasks but limited in their flexibility.'

-Churchland \& Sejnowski [1994: 7]-

'[...] un conjunto de sistemas interconectados con propósito específico, que son muy eficientes en la ejecución de sus tareas pero de muy limitada flexibilidad.'

La Facultad del Lenguaje es considerada, dentro de este emergente contexto de investigación, como uno de los 'sistemas con propósito específico' de que dispone el cerebro humano, nivelada en este sentido a otras facultades como la visión, la locomotricidad, etc. -véase Fodor [1983]-. La hipótesis atribuye al lenguaje, en consecuencia, cierta especificidad en cuanto a número, localización y morfología del tejido neural que le corresponde. La Facultad del Lenguaje se nos ofrece, por tanto, como un sistema cognitivo basado en un soporte de 'ingeniería natural', atributo que ciertamente no le va mal, pues a diferencia de los productos de la 'ingeniería artificial o cultural', sus mecanismos se nos presentan -al menos hasta ahora-como cerrados a cualquier género de revisión encaminado a mejorar su rendimiento -véase Churchland \& Sejnowski [1994: 8]-.

Esto no significa que la Lingüística trate de acaparar el estudio del soporte neural propio del lenguaje. No, la ciencia del lenguaje no es natural en este sentido. El territorio natural en el que debe concentrarse la Lingüística consiste en la facultad o módulo mental que 'emerge' de la interacción de los tejidos de ese sector del cerebro. Esta facultad se concreta en un conjunto de propiedades que el lingüista debe identificar en términos abstractos, tratando de discernir con precisión aquellos aspectos de las lenguas particulares que remiten de forma inmediata a la base natural, y diferenciándolos de aquellos otros que pudieran surgir de manipulaciones de carácter cultural. Queda trazada de este modo la distinción entre un núcleo y una periferia lingüísticos, expresión el primero de las limitaciones naturales que operan sobre la gama de lenguas particulares posibles, muestra la segunda de una cierta permeabilidad a los caprichos de la cultura. El objeto natural de la Lingüística se concreta en el sistema propiedades abstractas atribuible a toda lengua, el cual configura el dominio del módulo lingüístico de la mente humana.

La explicación de las propiedades de este módulo mental en términos de los tejidos y de la actividad somática realizada en los sectores cerebrales correspondientes cae en cambio fuera de la competencia de la Lingüística. Ahora bien, a la luz de la evolución de ciertos dominios de la ciencia en fechas no demasiado lejanas, empieza a cobrar crédito la idea de que la Lingüística, en tanto que estudio abstracto de ciertas propiedades de la mente, consiste en una disciplina de transición que prepara el terreno a una rama de la Neurociencia, que nos permitirá explicar ya las diferentes propiedades de las lenguas en términos de los mecanismos cerebrales en que se apoyan. Otero [1991] considera que la misión del enfoque naturalista sobre el lenguaje huma- 
no puede considerarse comparable a la que correspondió a la química decimonónica con relación a la ulterior cristalización de los enfoques fisicistas de la disciplina. Señala Otero lo siguiente:

'[...] we might think of nineteenth century chemistry as a study of the properties of the unkown physical mechanisms, framed in terms of such abstract notions as chemical elements, valence, organic molecules, the Periodic Table, and so on, this abstract study setting the stage for subsequent inquiry into the 'more fundamental' entities that exhibit the properties that are formulated at the abstract level of inquiry.'

-Otero [1991: 37]-

'[...] podríamos pensar acerca de la química del siglo XIX como el estudio de las propiedades de mecanismos físicos desconocidos, elaborado en términos de nociones tan abstractas como elementos químicos, valencia, moléculas orgánicas, la Tabla Periódica, etc., estudio abstracto que establece el soporte para la investigación posterior relativa a entidades 'más elementales' que exhiben las propiedades formuladas en el nivel abstracto de investigación’.

Otero señala además que esta misión es crucial e inexcusable para la disciplina, pues en su opinión:

'It is simply not possible to identify the specific features of the physical mechanisms of the brain that realize the properties postulated in the abstract study without first discovering what these properties are.'

-Otero [1991: 40]-

'Es sencillamente imposible identificar los rasgos específicos de los mecanismos físicos del cerebro que desarrollan las propiedades postuladas en el estudio abstracto sin descubrir antes cuáles son esas propiedades.'

Significa esto que la Lingüística representa un eslabón irrenunciable en el curso de una de las empresas más ambiciosas de las Ciencias de la Naturaleza: caracterizar desde un punto de vista físico la dimensión sin duda más característica del cerebro humano. Significa también que cualquier avance parcial lo suficientemente fundado en el estudio físico del cerebro deberá actuar también en el sentido de marcar pautas y de condicionar la forma (y la complejidad) de las hipótesis que los lingüistas sigan formulando a propósito de las propiedades abstractas del lenguaje humano. Como nos recuerda del Teso [1990: 32-33], no todas las hipótesis que 'circulan' en el ambiente de una determinada disciplina pueden considerarse 'principios' de esa disciplina: quiere decirnos que, a menudo, la explicación de un fenómeno en un determinado campo está parcialmente condicionada por fenómenos pertenecientes a otros campos. Es conveniente, por tanto, que el lingüista trabaje ya atento a los estímulos que pueda 
recibir de la investigación física del cerebro, una ciencia indiscutible y exhaustivamente natural. Y aquí se nos revela también un aspecto del carácter natural del estudio del lenguaje: gran parte de las disciplinas a las que los lingüistas deberán prestar en adelante una especialísima atención para conducir su trabajo son disciplinas con una indiscutible base natural, y la catalogación de una ciencia es, en buena medida, una cuestión de 'entorno' de trabajo.

2.2. Disonancia e imperceptibilidad. Me ocuparé a continuación de un par de atributos característicos de las modernas ciencias naturales. Interesa hacerlo porque algunos de ellos son vistos como defectos cuando se manifiestan asociados a una disciplina de contenido cultural, ignorando que son, en buena medida, el motor de los grandes avances apreciados en el contexto de las ciencias de la naturaleza. Si el estudio del lenguaje es también, como se razona aquí, una disciplina de contenido natural, lo preocupante sería, más bien, que no los viéramos encarnarse en ella. El primero de estos temas es el llamado problema de la 'disonancia con la intuición'; el segundo, el de la incorporación en el análisis de entidades no directamente perceptibles.

2.2.1. La disonancia con la intuición. Un rasgo muy característico de la investigación en el terreno de las ciencias naturales consiste en un cierto desprecio a los dictados de la intuición y del sentido común. El tema de la 'disonancia con la intuición' ha sido, de hecho, explícitamente tratado en el arranque de muchas investigaciones de contenido natural correspondientes a disciplinas más o menos jóvenes -véase, por ejemplo, los ya citados Churchland y Sejnowski [1994: 2], con relación a la investigación neurocomputacional, y Otero [1984: 42-49], ya a propósito de la Lingüística-. Esto es indicativo de que se trata de una especie de 'trauma' habitual en cualquier programa de investigación que cobra conciencia de su estatuto de ciencia de la naturaleza. Churchland y Sejnowski señalan lo siguiente:

'From the revolutions wrought by Copernicus, Galileo, Darwin, and Einstein, it is all too apparent that 'intuition dissonance' is a poor indicator of truht; it is a good indicator only of how one idea sits with well-favored others. Establising truth or probability requires rather more.'

-Churchland \& Sejnowski [1992: 2]-

'Desde las revoluciones de Copérnico, Galileo, Darwin y Einstein está bien claro que la 'disonancia con la intuición' en un pobre indicador de verdad; es sólo un buen indicador de cómo una idea encaja bien con otras con buena aceptación. El establecimiento de la verdad exige bastante más.'

Es importante considerar, además, que los resultados de una investigación científica no suelen tener siquiera el efecto de reemplazar nuestras opiniones de sentido común. Como dice Chomsky: 


\section{EL ESTUDIO DEL LENGUAJE COMO CIENCIA NATURAL}

'Nos es imposible salir de nuestra piel: nunca superaremos la ilusión de la luna o la visión del sol poniente [...].'

-Chomsky [1994: 95]-

Ahora bien, la no 'reemplazabilidad' no es prueba de debilidad de los enunciados científicos: es sólo indicativa de que sirven a otros fines y de que deben evaluarse atendiendo a criterios diferentes de su rendimiento en el curso más cotidiano de nuestras vidas. Dice también Chomsky:

'Si se plantea que el espacio es curvo, o que el mundo consiste en infinitas cuerdas unidimensionales en un espacio de diez dimensiones o en partículas sin masa, o que el tiempo tuvo un principio, o cualquier idea extraña que pueda aparecer mañana, la hipótesis tiene que se analizada por sus méritos, por muy ofensiva que pueda resultar para nuestras intuiciones de sentido común, que son, al fin y al cabo, intrascendentes.'

-Chomsky [1994: 95]-

Así pues, los enunciados de la intuición y los que resultan de la investigación metódica no compiten por los mismos espacios vitales. Por tanto, se evalúan alternativa y no simultáneamente: por ejemplo, la idea de la disposición interplanetaria de la que se sigue nuestra percepción de la salida y puesta del sol y la luna la aceptamos en la medida en que nos resulta útil para pautar el curso de cada jornada; en cambio, la idea de la disposición interplanetaria que resulta de la investigación astronómica la aceptamos en la medida en que facilita, por ejemplo, la llegada del hombre a los diferentes planetas.

Es hora de volver al lenguaje. Muchas de las explicaciones de la 'lingüística naturalista' se enfrentan claramente a las conceptualizaciones del lenguaje basadas en la intuición y en sentido común. Repasemos como ejemplo una de las explicaciones que han sido propuestas en el empeño por entender el tipo de regularidades interlingüísticas de las que nos ocupábamos en la sección 1.2. Atengámonos a los datos del español y del inglés como representantes de cada uno de los dos tipos lingüísticos que allí trazamos:

\section{Español}

a. __ Comerá en casa

b. Acudirá mi madre en mi lugar

c. ¿Quién dijiste que _ acudirá en tu lugar?

\section{Inglés}

a. *_ will eat at home

fut. comer en casa 
b. * will go my mother instead of me fut. ir mi madre en lugar de mí

c. * who did you say that _ will go instead of you? quién pas. tú decir que fut. ir en lugar de mí

Se ha señalado que la aparente omisión del sujeto en la oración (a.) del español es sólo una ilusión. En realidad, entre el sistema de propiedades que subyace a toda lengua se encuentra una que impone que toda oración debe tener saturada su 'posición de sujeto'. La peculiaridad del español en este sentido consistiría en que su léxico dispone de un elemento pronominal abstracto, es decir, no realizable desde el punto de vista fónico, que es posible insertar en esa posición, y la diferencia con el inglés en que esta lengua no tolera la inserción de ese mismo elemento en la posición de sujeto.

En principio, lo dicho da cuenta únicamente del contraste entre los ejemplos de tipo (a.) en cada una de las lenguas. La extensión de esta explicación a los ejemplos de tipo (b.) consiste en suponer que cuando el sujeto oracional se manifiesta pospuesto al verbo ocupa una posición semejante a la de los restantes complementos: por consiguiente, la posición preverbal (la 'posición de sujeto') estará sin ocupar, en contra de la disposición universal que rige al respecto. Para el español no hay mayor problema, porque está a su alcance la inserción del pronombre abstracto en la posición de sujeto; para el inglés, en cambio, la posposición del sujeto al verbo introduce en la oración un problema insoluble, ya que el pronominal abstracto no tiene cabida en la posición de sujeto.

Pasemos ahora a los ejemplos de tipo (c.), en los que se manifiesta la situación tal vez más complicada. Se asume, por razones en parte independientes al caso que aquí nos ocupa, que las palabras interrogativas se introducen inicialmente en la posición del entorno verbal que corresponde a las palabras no interrogativas alusivas al mismo tipo de información argumental. En (c.), por ejemplo, 'quién' y 'who' se insertan en la posición de sujeto de 'acudir' y de 'go', respectivamente, y son luego desplazadas a la cabecera de la oración, dejando un testigo abstracto en la posición de origen (una 'huella'). Teniendo esto en cuenta, supongamos que las posiciones de sujeto y de complemento no sólo son diferentes en términos estructurales, sino que además el verbo mantiene con cada una de ellas un tipo diferente de relación. En concreto, supongamos que la cohesión entre el verbo y los complementos es más estrecha que la cohesión entre el verbo y el sujeto, y admitamos además que el fundamento de esa mayor cohesión se encuentra en un tipo de vínculo o propiedad abstracta a la que llamaremos ' $\mathrm{R}$ '. La incorrección de la oración inglesa podría explicarse entonces como consecuencia de un requisito impuesto a las huellas (en adelante, 'h'), las cuales necesitan estar bajo el amparo de la propiedad ' $R$ ' para que los ejemplos en que aparecen resulten legítimos. Una representación de los entresijos del ejemplo (c.) del inglés conforme a los presupuestos establecidos sería la siguiente: 
* who $o_{1}$ did you say that $\left[\mathrm{h}_{1}\right.$ [will go instead of you $]$ ?

$\uparrow \neg \mathrm{R}\lrcorner$

En esta representación se da a entender que la huella que resulta del desplazamiento de 'who' queda fuera del alcance de la propiedad 'R', cuya fuente es el verbo, de modo que la oración resulta incorrecta.

La razón de que la oración equivalente sea en cambio correcta en español tiene que ver con la explicación del contraste entre los ejemplos del tipo (b.). Debido a que la permisividad de esta lengua a situar el pronombre abstracto en la posición de sujeto hace tolerable la presencia del argumento sujeto en una posición de complemento, podemos suponer que la palabra interrogativa se desplaza en español desde esta última posición. Se obtiene de este modo que la huella introducida por el desplazamiento cae bajo el alcance de la propiedad ' $R$ ' del verbo, de manera que su presencia en el entramado oracional no acarrea mayores problemas. Todo lo anterior se puede representar de la siguiente manera, donde 'pro' alude al pronominal abstracto:

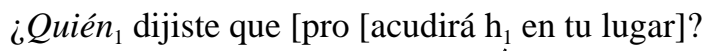

L R $\uparrow$

No cabe duda de que los análisis sugeridos no sustituyen en modo alguno nuestra percepción de los hechos del lenguaje, y que incluso para ciertos fines nos resultará más cómodo seguir expresándonos como si la estructura oracional no constara de otros elementos que aquellos que se desprenden de nuestra percepción más inmediata -es decir, como si se cifraran al margen de una estructura preprogramada de conocimiento lingüístico localizada en la mente del hablante-. Con todo, una hipótesis como la que he presentado de manera muy simplificada se acompaña de indudables 'méritos' -Chomsky, arriba-. En el caso revisado, la postulación de ciertas entidades y relaciones que sin duda retan a nuestro sentido común sirve para dar una explicación conjunta a fenómenos aparentemente diversos, pero que una observación atenta revela claramente relacionados entre sí en lenguas de filiación y localización muy diversas. Sólo en términos de este poder explicativo debe recibir evaluación la hipótesis, la cual entablará competencia con hipótesis con poderes explicativos afines, por mucho que 'disuenen' a nuestro sentido común, y nunca con planteamientos que no prevean explicación para los mismos fenómenos.

2.2.2. Lo no perceptible. Sin lugar a dudas, la piedra con la que tropieza una y otra vez el sentido común en la valoración de las explicaciones naturalistas radica en el sistema de propiedades no perceptibles en que tales explicaciones fundan parcialmente el 'conocimiento de una lengua'. Este aspecto del naturalismo lingüístico constituye sin embargo una premisa fundamental del enfoque, pues como expresa con precisión Otero: 
'The crucial fact is that behaviour involves use of knowledge, and the system of knowledge that underlies behavior is not realized in any direct or simple way in behaviour.'

-Otero [1991: 26]-

'El hecho crucial es que el comportamiento implica el uso de conocimiento, y que el sistema de conocimiento que subyace al comportamiento no es aprehensible de manera directa o simple del comportamiento.'

Lo que me interesa destacar en este último tramo de mi trabajo es la trascendental función que el manejo de este tipo de propiedades ha cumplido en la aproximación de la teoría lingüística a dominios que de otro modo no podríamos considerar más que como misterios para los que la disciplina no se encontraría ni remotamente preparada.

El curso de la investigación en el terreno de las ciencias de la naturaleza traza (en medida mucho más apreciable que en las disciplinas humanísticas más desarrolladas) un camino que va recortando terreno al 'misterio', reduciéndolo en primer término a la condición de 'problema(s)'. El problema, a diferencia del misterio, ya está sujeto a formulación racional; ha sido limitado y resulta, por tanto, compatible con el formato de nuestra técnica básica de explicación científica: la hipótesis. Por esta razón, la credibilidad de una hipótesis descansa básicamente en la posibilidad de enunciar el tipo de condiciones que demostrarían su falsedad. Ante un misterio, en cambio, del que por definición desconocemos el alcance, resulta imposible formular condiciones de las que se pueda decir que implican la falsedad de nuestras ideas al respecto -este tipo de ideas son, más bien, cuestiones de fe-.

Vayamos directamente al caso que nos interesa aquí. Señala correctamente del Teso [1991: 35-36] que la 'hipótesis de innatismo' -esto es, la postulación en el cerebro humano de una estructura de conocimiento lingüístico anterior a toda experienciasería totalmente gratuita en el contexto de la teoría estructuralista sobre el lenguaje humano, en el sentido de que no es posible especificar, sin salirse del marco conceptual de tal teoría, el tipo de observaciones que demostrarían la falsedad de la hipótesis. Digamos que dada la potencia de resolución de problemas alcanzada por la teoría estructuralista, cuestiones como la del innatismo caen del lado de lo que cuenta, frente a esa teoría, como 'misterio'. Es importante notar que el carácter de misterio -vinculado aquí a la imposibilidad de formular 'condiciones de falsación/verificación'- cuenta para el conjunto de la cuestión, de modo que tan gratuita será en este marco la 'hipótesis del innatismo' como la 'hipótesis de la tabula rasa', por más que ésta última haya sido incorporada, casi como estandarte, en muchas formulaciones estructuralistas de las propiedades del lenguaje humano. Por ejemplo, entre los universales que Hockett [1963: 11] atribuye al lenguaje frente a los sistemas de comunicación animal figura el siguiente:

'2.12. Tradition. The conventions of language are passed down by teaching and learning, not through the germ plasm.' 
'2.12. Tradición. Las convenciones del lenguaje se transmiten mediante la enseñanza y el aprendizaje, no a través del plasma germinal.'

Es tal vez cierto que nuestra propia constitución biológica impone topes a la indagación de carácter científico. Chomsky [1994: 99-103], por ejemplo, sostiene que toda especie animal tiene vedada la comprensión de ciertos aspectos del mundo natural, en función de las cualidades de sus equipamientos cognitivos -para una exposición más extensa de esta idea véase toda la Segunda Parte de Chomsky [1975]-. Tales aspectos contarán, por tanto, como los 'misterios absolutos' a los que se enfrenta cada una de las especies. Sin embargo, mucho antes de llegar al 'más allá' representado por esa extensión de misterio, ha de existir aún una extensión de 'misterios provisionales', es decir, de misterios susceptibles de ser reducidos a la condición de 'problemas'. Podemos decir que el 'misterio absoluto' evidencia los límites de nuestra capacidad para conocer; el 'misterio provisional' simplemente indica las limitaciones propias de las herramientas intelectuales -hipótesis- desarrolladas en virtud de tal capacidad en un determinado momento de la historia. Avanzar por el terreno de los misterios provisionales exige, básicamente, una actitud de desprendimiento y saludable desconfianza hacia las hipótesis heredadas, donde tal vez entre en juego, de manera crucial, el no dejarse impresionar por el efecto ya comentado de 'disonancia con la intuición'.

Volvamos a la cuestión del innatismo. El enfoque naturalista sobre el lenguaje humano ha conseguido reducir la cuestión al rango de problema porque ha comenzado a formular hipótesis sobre las propiedades de esa estructura predeterminada del cerebro humano, especializada en tareas lingüísticas, que se acompañan de 'condiciones de falsación'. Esto se ha conseguido, en buena medida, gracias a la postulación de 'propiedades no inmediatamente perceptibles' que parecen en efecto guiar la conformación de los mensajes lingüísticos: en la medida en que estas propiedades se revelan operativas a efectos independientes de la cuestión misma del innatismo, sirven de apoyo a la idea de que ciertos aspectos del conocimiento de las lenguas se fijan completamente al margen de los datos ofrecidos por el entorno durante el período de adquisición de la lengua.

Recordemos solamente dos de los elementos abstractos introducidos en el apartado anterior: la entidad 'pro' y la relación 'R'. Ya en ese apartado se pudo ver que su operatividad se extiende a una extensa gama de fenómenos y construcciones; es decir, que no se limitan a cubrir ciertos espacios en la descripción -o transcripción abstracta- de fenómenos o construcciones específicos, sino que pueden entenderse como fragmentos del conocimiento que un hablante despliega en la conformación de una variada gama de construcciones. Además de los casos vistos más arriba, en las lenguas en que se da la posibilidad de introducir 'pro' en la posición de sujeto se predice, por ejemplo, que en las oraciones con verbos de tipo impersonal no habrá sujetos léxicamente manifestados; en cambio, en las lenguas en que esa posibilidad no se consuma, 
se predice la obligatoriedad de insertar sujetos léxicos expletivos -esto es, carentes de todo contenido referencial-. Ambas predicciones se cumplen:

\section{Español}

a. __ Llueve mucho

b. __ Parece que hay bullanga

Inglés

a. It rains a lot $\backslash *$ _ Rains a lot

b. There seems to be a riot $\backslash *$ _ Seems to be a riot

La influencia de la relación ' $\mathrm{R}$ ' en un caso distinto al ofrecido más arriba se puede apreciar en el fenómeno de clitización de 'ne' en italiano, estudiada por Belleti y Rizzi [1981]. Fijémonos en el siguiente contraste:

\section{Italiano}

a. ne-ho visto molti de ellos he visto a muchos 'He visto a muchos de ellos'
b. * molti ne sono intelligenti muchos de ellos son inteligentes

La diferencia entre el ejemplo (a.), correcto, y el ejemplo (b.), incorrecto, reside en que en el primer caso el clítico se relaciona con el complemento verbal, y en el segundo caso con el sujeto. Lo podemos representar situando de nuevo una entidad abstracta - una 'huella' - en cada uno de esos enclaves estructurales, dando a entender que la formación de este tipo de ejemplos implica de algún modo a la operación de movimiento de constituyente de la que ya se habló en el caso de la formación de preguntas. Los análisis correspondientes serán los que siguen:

a. [ne $\mathrm{i}_{\mathrm{i}}$ ho visto [molti $\left.\mathrm{h}_{\mathrm{i}}\right]$ ]

$\llcorner\mathrm{R}$

b. * [[molti $\left.\mathrm{h}_{\mathrm{i}}\right]\left[\right.$ ne $_{\mathrm{i}}-$-sono intelligenti $\left.]\right]$

$\uparrow \neg \mathrm{R} \_$

El problema de (b.) consiste, por tanto, en que la huella que postulamos en el ejemplo cae fuera del ámbito de influencia de la relación ' $R$ '.

En todos los casos que hemos ido viendo se aprecia que el hablante hace espontáneamente uso de un cierto tipo de conocimiento no directamente identificable con las peculiaridades más superficiales de las muestras de su comportamiento. En la medida en que este género de atributos se revelan operativos en la fijación de los límites de la gra- 
maticalidad -desde un punto de vista intralingüístico- y los de la variación -desde un punto de vista interlingüístico-, apuntalan la hipótesis de que los seres humanos 'tienen conocimiento' de un sistema de elementos cognitivos abstractos al margen de su experiencia como 'hablantes-oyentes', sistema que sin duda configura una parcela especialmente característica del 'patrimonio natural' de la especie.

\section{Conclusión}

A lo largo de este trabajo he intentado dar apoyo a la idea de que el lenguaje es, ante todo, un componente natural más del universo. Debe quedar claro que esta idea no es incompatible con el reconocimiento de que se trata al mismo tiempo de una facultad mental que se exterioriza en objetos enormemente permeables a ciertos impulsos de la cultura. Con todo, si las ideas que he tratado de apoyar aquí van por el buen camino, el estudio de los efectos de la cultura sobre las lenguas particulares deberá considerarse como un tipo de investigación básicamente subordinado al de las fuentes naturales que, como decíamos al inicio del trabajo, limitan su espacio y condicionan su misma posibilidad de ser.

- Una primera versión de este trabajo fue impartida como conferencia en el Departamento de Filoloxía Española, Teoría da Literatura e Lingüística Xeral de la Universidade de Santiago de Compostela, el 3 de mayo de 1995. Deseo dar las gracias a la audiencia de esa conferencia, y muy especialmente a los profesores Milagros Fernández Pérez y Víctor Manuel Longa.

\section{Bibliografía}

BELLETI, A. \& LUIGI R. (1981), The Syntax of 'Ne': Some Theorical Implications, The Linguistic Review, v. 1.

BICKERTON, D. (1990), Language and Species, University of Chicago Press [traducción castellana de Miguel Angel Valladares, Lenguaje y Especies, Madrid: Alianza Universidad, 1994].

CHOMSKY, N. A. (1975), Reflections on Language, New York, Pantheon Books [traducción castellana de J.A. Argente y J.M. Nadal, Reflexiones sobre el Lenguaje, Barcelona: Ariel, 1979].

(1994), Creación y Cultura; Política y Cultura a Finales del Siglo XX, Barcelona: Ariel [traducción castellana de José Manuel Álvarez Flórez de una serie de conferencias organizadas por la Cátedra Ferrater Mora de Pensamiento Contemporáneo de la Universitat de Girona].

CHURCHLAND, P. S. \& TERRENCE, J. S. (1994) The Computational Brain, Cambridge (MA), The MIT Press. 
COSERIU, E. (1978), Los Universales del Lenguaje (y los Otros); Gramática, Semántica y Universales. Estudios de Lingüística Funcional, Madrid: Gredos.

FERNANDEZ DE CASTRO, F. (1993), La Continuidad del Naturalismo Lingüístico, Revista Española de Lingüística, v. 23.

FODOR, J. A. (1983), The Modularity of Mind, Cambridge (MA), The MIT Press.

HOCKETT, C. F. (1963), The Problem of Universals in Language; in J.H. Greenberg (ed.) Universals of Language, Cambridge (MA), The MIT Press.

Jaeggli, Osvaldo \& Kenneth J. Safir (eds.)

(1989), The Null Subject Parameter, Dordrecht, Kluwer.

MARTINET, A. (1960), Éléments de Linguistique Générale, Paris, Librairie Armand Colin [traducción castellana de Julio Calonge Ruiz, Elementos de Lingüística General, Madrid: Gredos, 1965 (citado por la tercera edición de 1984)].

OTERO, C. P. (1984) La Revolución de Chomsky. Ciencia y Sociedad, Madrid: Tecnos.

(1991), The Cognitive Revolution and the Study of Language: Looking Back to See Ahead; in H. Campos \& F. Martínez-Gil (eds.) Current Studies in Spanish Linguistics, Washington, D.C., Georgetown University Press.

PRIETO, L. J. (1973), Prólogo a la Edición Española, en N.S. Trubetzkoy, Principios de Fonología, Madrid, Cincel.

RIZZI, L. (1989), The New Comparative Syntax: Principles and Parameters of Universal Grammar, Cahiers Ferdinad de Saussure, v. 43.

DE SAUSSURE, F. (1915), Cours de Linguistique Générale [traducción castellana de Amado Alonso, Curso de Lingüística General, Madrid: Alianza Universidad Textos, 1983; según la edición critica de Tulio de Mauro].

SMITH, N. \& IANTHI M. T. (1991), Linguistic Modularity? A Case Study of a 'Savant' Linguist. Lingua, v. 84.

DEL TESO, E. (1990), Gramática General, Comunicación y Partes del Discurso, Madrid: Gredos. 